\title{
Supporting Information: Molecular Calculation of the Critical Parameters of Classical Helium
}

\author{
Richard A. Messerly, ${ }^{\dagger}$ Navneeth Gokul, ${ }^{\ddagger}$ Andrew J. Schultz, ${ }^{\ddagger}$ David A. Kofke, ${ }^{\ddagger}$ \\ and Allan H. Harvey*, \\ $\dagger$ †pplied Chemicals and Materials Division, National Institute of Standards and \\ Technology, 325 Broadway, Boulder, CO 80305 \\ $\ddagger$ Department of Chemical and Biological Engineering, University at Buffalo, The State \\ University of New York, Buffalo, NY 14260-4200, USA \\ ФPresent address: Biosciences Center, National Renewable Energy Laboratory, 15013 \\ Denver W Pkwy, Golden, CO 80401 \\ E-mail: allan.harvey@nist.gov
}




\section{Supporting Information for Gibbs Ensemble Simulation}

\section{Finite-size effects}

Figure S1 compares GEMC results obtained with different number of molecules $(N)$ and cut-off distances $\left(r_{\text {cut }}\right)$. Results are statistically indistinguishable for $N=800$ to 2800 and for $r_{\text {cut }}=1.0 \mathrm{~nm}$ to $1.8 \mathrm{~nm}$.

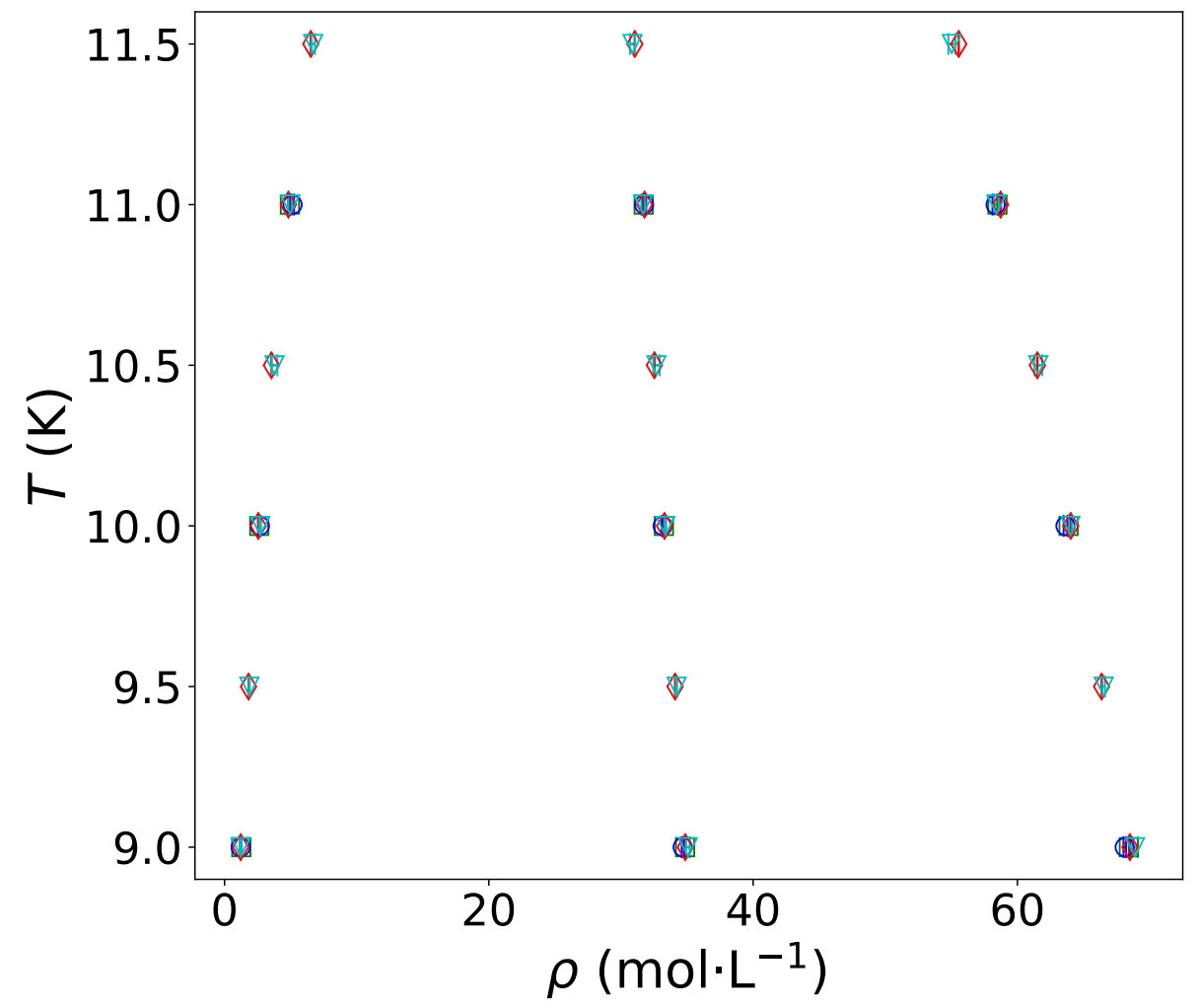

$$
\begin{aligned}
& N=2800, r_{\text {cut }}=1.8 \mathrm{~nm} \quad \mapsto \quad N=1400, r_{\text {cut }}=1.0 \mathrm{~nm} \\
& \text { 머 } N=2800, r_{\text {cut }}=1.4 \mathrm{~nm} \quad \text { it } N=800, r_{\text {cut }}=1.0 \mathrm{~nm}
\end{aligned}
$$

Figure S1: Finite-size effects at subcritical conditions. 


\section{Vapor phase compressibility factor}

Figure S2 plots the vapor phase compressibility factor $\left(Z_{\text {vap }}^{\text {sat }}\right)$. The physically realistic trend (converging smoothly to 1 at low $T^{\text {sat}}$ ) helps validate the $\rho_{\text {vap }}^{\text {sat }}$ and $P^{\text {sat }}$ GEMC values.

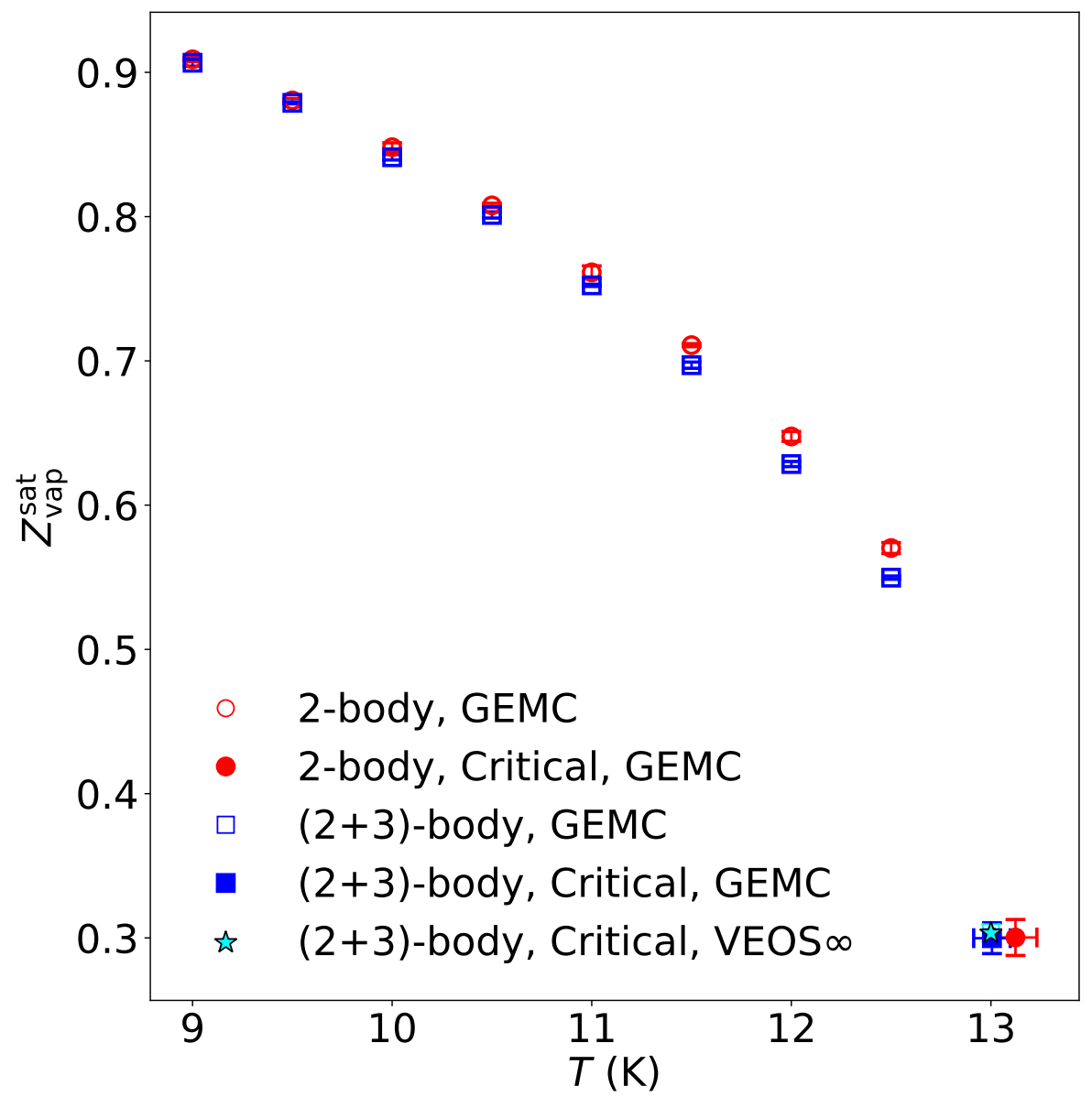

Figure S2: Vapor phase compressibility factor with respect to temperature. Data correspond to the systems presented in the manuscript. 
Table S1: VEOS6 saturation pressures for two- and (2+3)-body classical He obtained by using $T^{\text {sat }}$ and $\rho_{\text {vap }}^{\text {sat }}$ from Table 4 in the article. Expanded uncertainties $(k=2)$ are propagated from the stochastic uncertainty in the virial coefficients, and do not include any effects of inaccuracy in the two- and three-body potentials.

\begin{tabular}{|c|c|c|c|}
\hline$T^{\text {sat }}(\mathrm{K})$ & $\rho_{\text {vap }}^{\text {sat }}\left(\mathrm{mol} \cdot \mathrm{L}^{-1}\right)$ & $P^{\text {sat }}(\mathrm{MPa})$ & $Z_{\text {vap }}^{\text {sat }}$ \\
\hline \multicolumn{4}{|c|}{ Two-body } \\
\hline 9 & 1.222 & $0.083291700(4)$ & $0.91086500(4)$ \\
9.5 & 1.816 & $0.126262000(16)$ & $0.88023300(11)$ \\
10 & 2.55 & $0.17970000(6)$ & $0.8475660(3)$ \\
10.5 & 3.55 & $0.2502520(3)$ & $0.8074680(8)$ \\
11 & 4.83 & $0.3368260(10)$ & $0.762485(2)$ \\
11.5 & 6.53 & $0.443146(4)$ & $0.709745(6)$ \\
12 & 8.88 & $0.572217(17)$ & $0.645851(19)$ \\
12.5 & 12.15 & $0.72138(8)$ & $0.57127(6)$ \\
\hline \multicolumn{4}{|c|}{$(2+3)-$ body } \\
\hline 9 & 1.29 & $0.087455000(5)$ & $0.90598000(6)$ \\
9.5 & 1.844 & $0.127963000(19)$ & $0.87854900(13)$ \\
10 & 2.66 & $0.18607400(8)$ & $0.8413360(4)$ \\
10.5 & 3.66 & $0.2562940(3)$ & $0.8021110(10)$ \\
11 & 5.06 & $0.3482040(13)$ & $0.752413(3)$ \\
11.5 & 6.9 & $0.458976(6)$ & $0.695679(8)$ \\
12 & 9.43 & $0.59124(3)$ & $0.62841(3)$ \\
12.5 & 13.08 & $0.74459(13)$ & $0.54773(10)$ \\
\hline
\end{tabular}




\section{Software comparison}

Figure S3 compares the vapor-liquid coexistence curves obtained with two different software packages, namely, Cassandra and Towhee. For a fair comparison, simulations were performed with the same number of molecules $(N=2800)$ and long-range cut-off distance $\left(r_{\text {cut }}=1.4\right.$ $\mathrm{nm})$. The Towhee results were obtained by utilizing the native tabulated potential function. Note that long-range tail corrections are not available in Towhee for a tabulated potential. The good agreement between the Cassandra (with tail corrections) and Towhee (without tail corrections) indicates that long-range tail corrections are negligible beyond $1.4 \mathrm{~nm}$. Furthermore, Towhee does not allow for virial pressure calculations with tabulated potentials. For this reason, we do not compare the vapor pressures computed with the two software packages.

\section{Supporting Information for VEOS}

\section{Critical Parameter Extrapolation}

In order to obtain the $n=\infty$ predictions for the helium critical parameters, the properties for each $n$ are reduced by the Lennard-Jones (LJ) critical parameters of the respective order. Relevant data are presented in Table S2. A bootstrap analysis is used to estimate the uncertainties in the critical properties, and their correlation with respect to $n$. These statistics are used in a generalized linear regression of the critical-parameter ratios with respect to $1 / n$. For the purposes of the fit, the LJ critical parameters are assumed to be exact. The correlations and uncertainties required for the constructing the linear fit of $T_{\mathrm{c}}$, $P_{\mathrm{c}}$ and $\rho_{\mathrm{c}}$ are recorded in Tables $\mathrm{S} 3, \mathrm{~S} 4$ and $\mathrm{S} 5$ respectively. This fit is then used to obtain a prediction for the ratio at $1 / n \rightarrow 0$ by extrapolation and the result is multiplied by the simulation value of the LJ critical parameter to obtain a prediction for the helium critical parameter in the limit. 


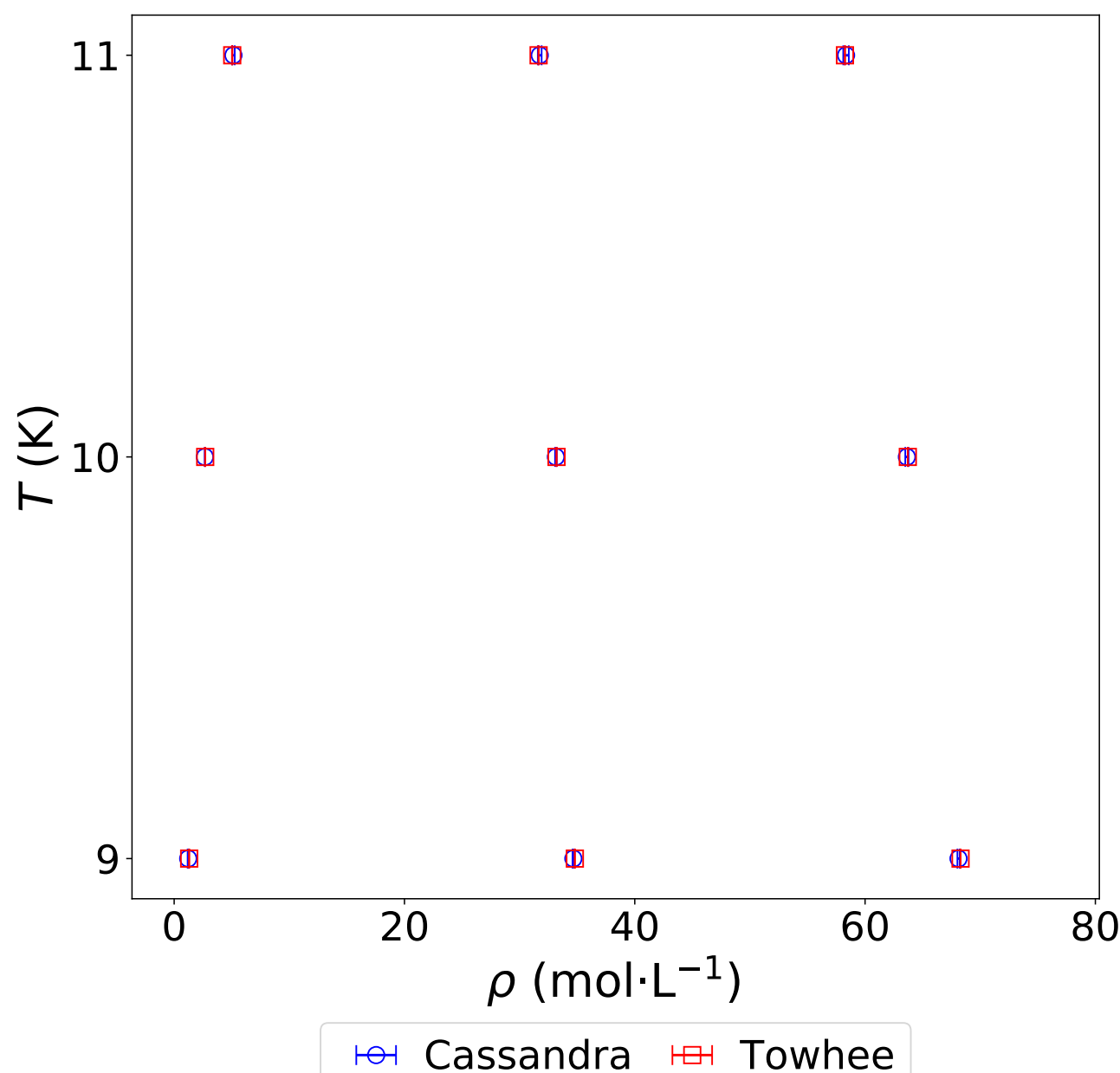

Figure S3: Comparison of vapor-liquid coexistence curve between Cassandra and Towhee. All simulations were performed with the two-body potential, $N=2800$, and $r_{\text {cut }}=1.4 \mathrm{~nm}$.

The fit and the points used to construct the fit are multiplied by the simulation value of the Lennard-Jones critical parameter and are shown in Figures S4, S5 and S6 for $T_{\mathrm{c}}, P_{\mathrm{c}}$ and $\rho_{\mathrm{c}}$, respectively. 
Table S2: Critical parameters of (2+3)-body classical helium and Lennard-Jones (LJ) fluid from VEOS $n$. LJ properties are in units such that $\sigma$ and $\epsilon / k_{\mathrm{B}}$ are each unity. Numbers in parentheses are expanded uncertainties $(k=2)$ in the last digit.

\begin{tabular}{|c|c|c|c||c|c|c|c|}
\hline \hline & \multicolumn{3}{|c||}{ He (2+3)-Body } & & \multicolumn{3}{c|}{ LJ } \\
\hline$n$ & $T_{\mathrm{c}} / \mathrm{K}$ & $P_{\mathrm{c}} / \mathrm{MPa}$ & $\rho_{\mathrm{c}} / \mathrm{mol} \cdot \mathrm{L}^{-1}$ & $n$ & $T_{\mathrm{c}}$ & $P_{\mathrm{c}}$ & $\rho_{\mathrm{c}}$ \\
\hline 3 & $14.09240(8)$ & $1.23325(2)$ & $31.5758(4)$ & 3 & 1.44528 & 0.177899 & 0.36927 \\
4 & $12.7416(4)$ & $0.86147(10)$ & $23.142(2)$ & 4 & 1.29918 & 0.122291 & 0.267471 \\
5 & $12.682(6)$ & $0.8464(16)$ & $22.73(4)$ & 5 & 1.29057 & 0.11933 & 0.260968 \\
6 & $13.08(9)$ & $0.97(3)$ & $28(6)$ & 6 & 1.31867 & 0.13023 & 0.293664 \\
7 & $13.2(2)$ & $1.02(8)$ & $29(3)$ & 7 & 1.32234 & 0.13186 & 0.300884 \\
\hline & & & & $\operatorname{Sim}^{1}$ & $1.3128(16)$ & $0.1274(13)$ & $0.316(4)$ \\
\hline
\end{tabular}


Table S3: Correlations and expanded uncertainties $(k=2)$ for $T_{\mathrm{c}}$ as computed by VEOSn. Values are computed using bootstrap resampling and reflect the effect of uncertainty in the virial coefficients.

\begin{tabular}{|c|ccccc|c|}
\hline$n$ & 3 & 4 & 5 & 6 & 7 & Unc. $T_{\mathrm{c}} / \mathrm{K}$ \\
\hline 3 & 1 & 0.136844 & 0.0504166 & 0.0649895 & 0.0448385 & 0.0000835983 \\
4 & 0.136844 & 1 & 0.125524 & -0.0434103 & 0.00574796 & 0.00042768 \\
5 & 0.0504166 & 0.125524 & 1 & 0.135249 & -0.0126583 & 0.00623466 \\
6 & 0.0649895 & -0.0434103 & 0.135249 & 1 & 0.374519 & 0.0896953 \\
7 & 0.0448385 & 0.00574796 & -0.0126583 & 0.374519 & 1 & 0.218812 \\
\hline
\end{tabular}

$T_{\text {c Fit: } 1.313(9.90358-0.384572 / n)}$

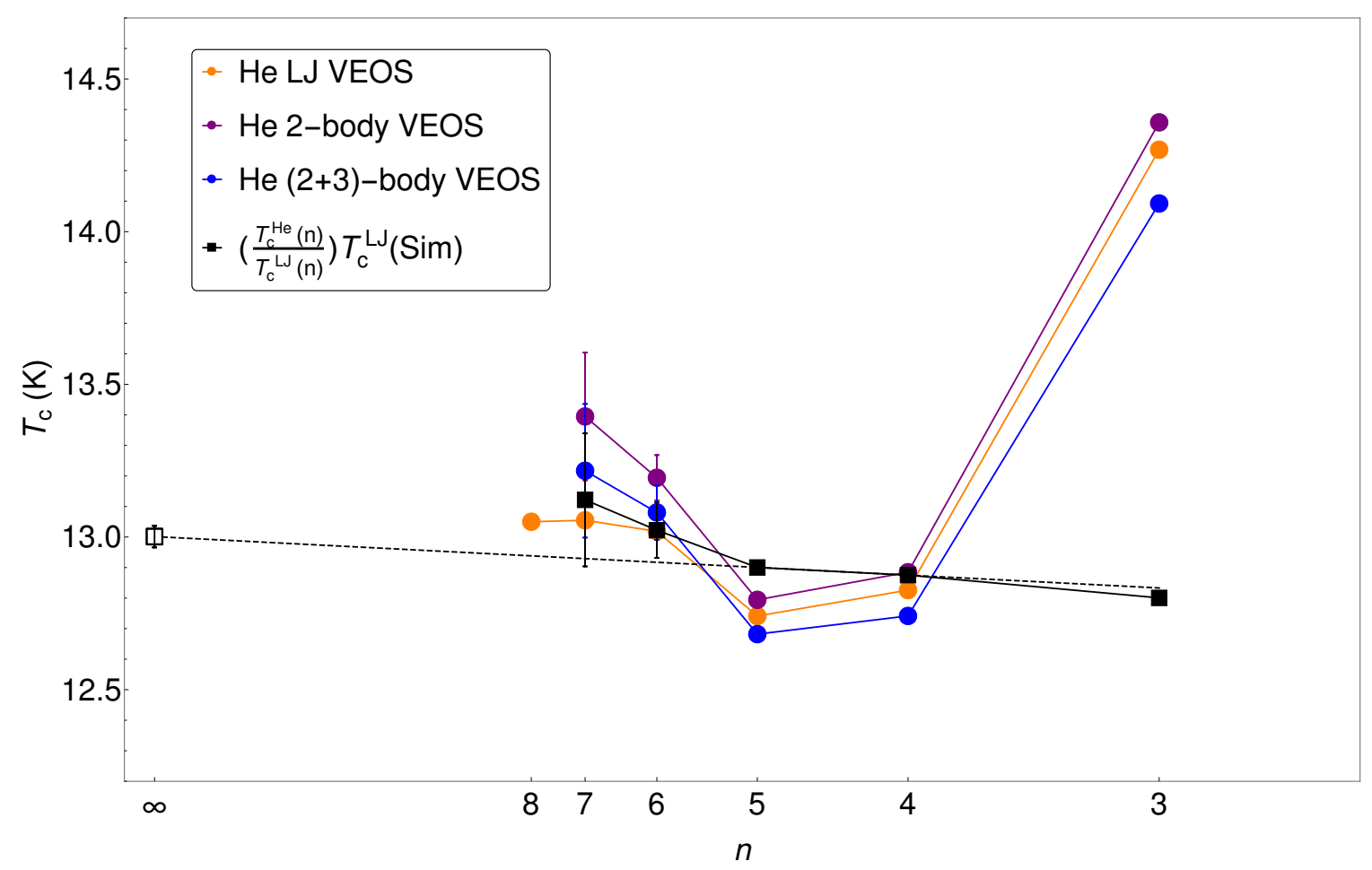

Figure S4: Critical temperature as given by VEOSn. Values are shown for the classical helium model (up to $n=7$ ) both with and without the three-body potential, and for the simple Lennard-Jones (LJ) model (up to $n=8$ ), using coefficients reported in previous work ${ }^{2-4}$ and using LJ size and energy parameters ${ }^{5} \epsilon / k_{\mathrm{B}}=9.8725 \mathrm{~K}$ and $\sigma=0.25238 \mathrm{~nm}$. The dashed line is a linear fit of the black squares (excluding $n=3$ ), which is the LJ-assisted extrapolation construct described in the text; the $n \rightarrow \infty$ extrapolated value is shown by the open black square. Error bars represent expanded $(k=2)$ uncertainty estimates. This is the same as the figure presented in the main text. 
Table S4: Correlations and expanded uncertainties $(k=2)$ for $P_{\mathrm{c}}$ as computed by VES $n$. Values are computed using bootstrap resampling and reflect the effect of uncertainty in the virial coefficients.

\begin{tabular}{|c|ccccc|c|}
\hline$n$ & 3 & 4 & 5 & 6 & 7 & Unc. $P_{\mathrm{c}} / \mathrm{MPa}$ \\
\hline 3 & 1 & 0.111452 & 0.0480238 & 0.0657252 & 0.0469009 & 0.0000218795 \\
4 & 0.111452 & 1 & 0.117564 & -0.0503512 & 0.00581682 & 0.0000973835 \\
5 & 0.0480238 & 0.117564 & 1 & 0.117093 & -0.0156593 & 0.00155834 \\
6 & 0.0657252 & -0.0503512 & 0.117093 & 1 & 0.341353 & 0.0318824 \\
7 & 0.0469009 & 0.00581682 & -0.0156593 & 0.341353 & 1 & 0.0763665 \\
\hline
\end{tabular}

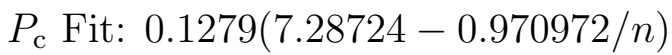

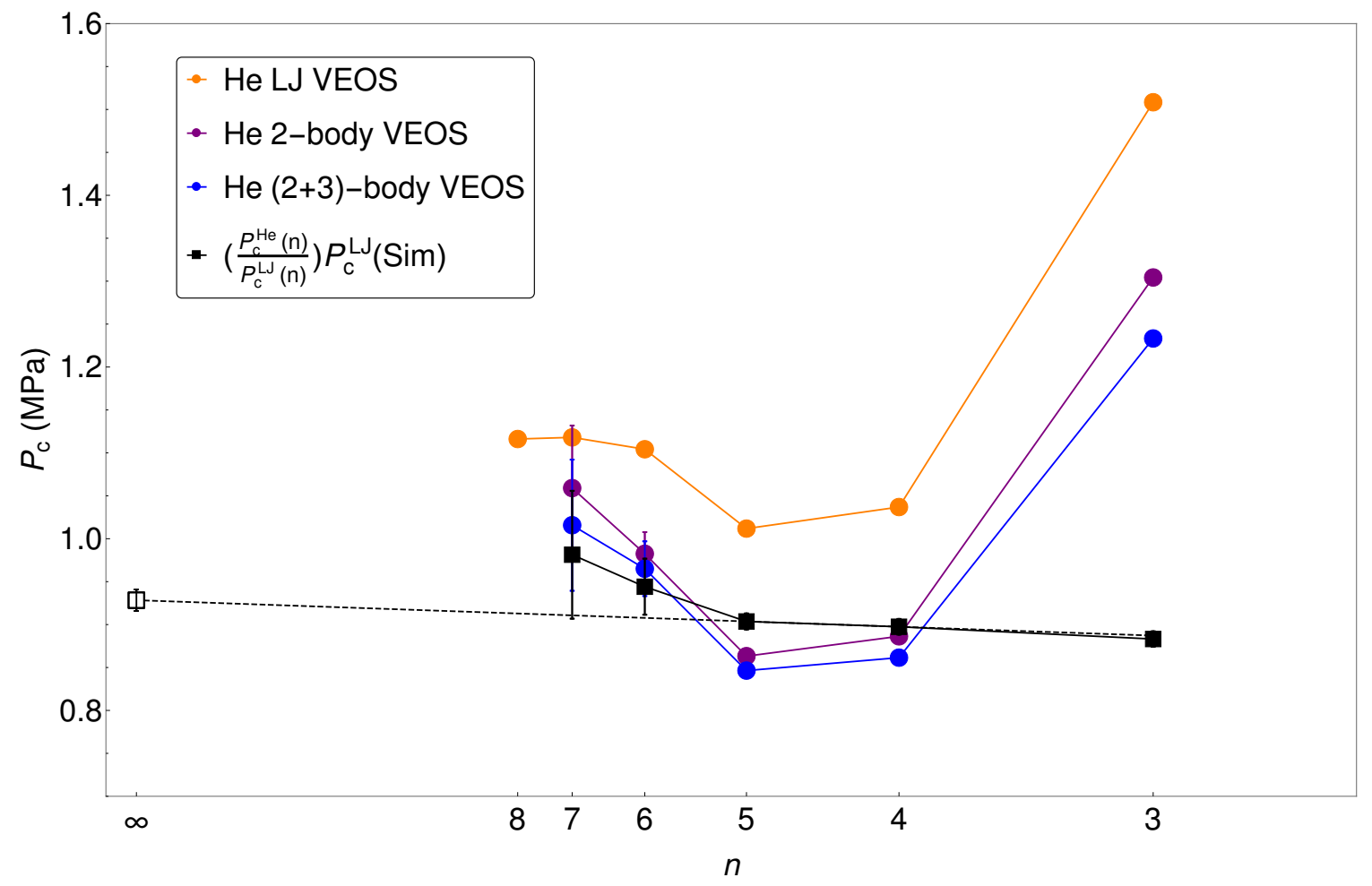

Figure S5: Same as Fig. S4, but for the critical pressure, $P_{\mathrm{c}}$. 
Table S5: Correlations and expanded uncertainties $(k=2)$ for $\rho_{\mathrm{c}}$ as computed by VEOS $n$. Values are computed using bootstrap resampling and reflect the effect of uncertainty in the virial coefficients.

\begin{tabular}{|c|ccccc|c|}
\hline$n$ & 3 & 4 & 5 & 6 & 7 & Unc. $\rho_{\mathrm{c}} / \mathrm{mol} \cdot \mathrm{L}^{-1}$ \\
\hline 3 & 1 & 0.0820036 & 0.0453338 & 0.0323436 & 0.0932722 & 0.000372884 \\
4 & 0.0820036 & 1 & 0.103834 & 0.00856657 & -0.0855269 & 0.00210352 \\
5 & 0.0453338 & 0.103834 & 1 & 0.110533 & -0.030441 & 0.042164 \\
6 & 0.0323436 & 0.00856657 & 0.110533 & 1 & 0.0786581 & 5.66912 \\
7 & 0.0932722 & -0.0855269 & -0.030441 & 0.0786581 & 1 & 2.61644 \\
\hline
\end{tabular}

$\rho_{\mathrm{c}}$ Fit: $0.317(89.5689-12.181 / n)$

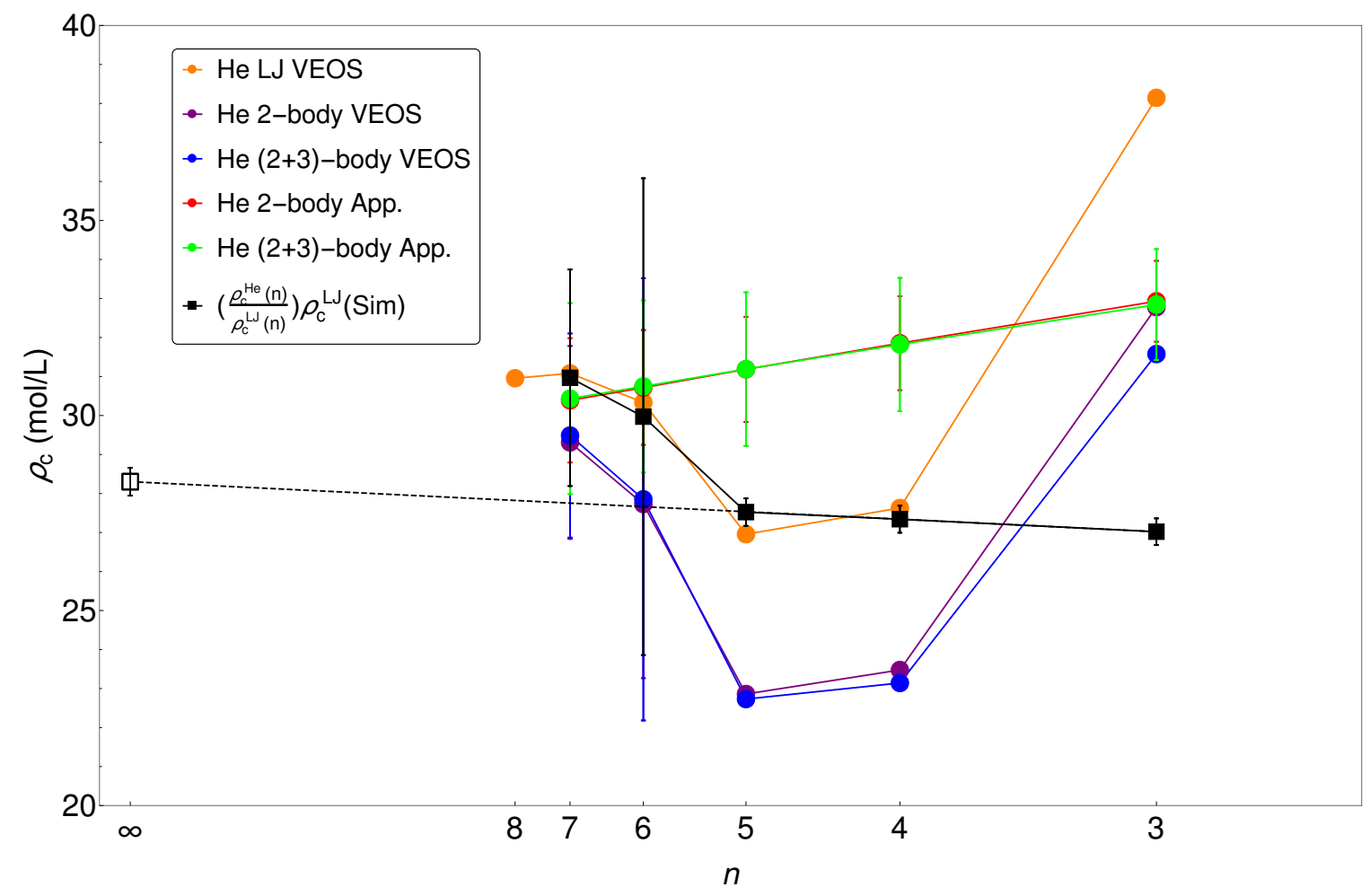

Figure S6: Same as Fig. S4, but for the critical density, $\rho_{\mathrm{c}}$; here, $n=3$ point is included in linear fit. Additionally, lines are presented showing the density as computed from the critical-isotherm approximant, ${ }^{6}$ using $T_{\mathrm{c}}$ and $P_{\mathrm{c}}$ for $n=6$. 


\section{Literature Cited}

(1) Dinpajooh, M.; Bai, P.; Allan, D. A.; Siepmann, J. I. Accurate and precise determination of critical properties from Gibbs ensemble Monte Carlo simulations. J. Chem. Phys. 2015, 143, 114113.

(2) Schultz, A. J.; Kofke, D. A. Sixth, seventh and eighth virial coefficients of the LennardJones model. Mol. Phys. 2009, 107, 2309-2318.

(3) Schultz, A. J.; Barlow, N. S.; Chaudhary, V.; Kofke, D. A. Mayer Sampling Monte Carlo calculation of virial coefficients on graphics processors. Mol. Phys. 2013, 111, 535-543.

(4) Feng, C.; Schultz, A. J.; Chaudhary, V.; Kofke, D. A. Eighth to sixteenth virial coefficients of the Lennard-Jones model. J. Chem. Phys. 2015, 143, 044504.

(5) Weaver, A. B.; Alexeenko, A. A. Revised Variable Soft Sphere and Lennard-Jones Model Parameters for Eight Common Gases up to 2200 K. J. Phys. Chem. Ref. Data 2015, 44, 023103 .

(6) Barlow, N. S.; Schultz, A. J.; Kofke, D. A.; Weinstein, S. J. Critical Isotherms from Virial Series Using Asymptotically Consistent Approximants. AIChE J. 2014, 60, 3336-3349. 